Research Article

\title{
Bounds on graph energy and Randić energy
}

\section{Ş. Burcu Bozkurt Altındağ*}

Yenikent Kardelen Konutları, Selçuklu, 42070 Konya, Turkey

(Received: 14 June 2021. Received in revised form: 24 June 2021. Accepted: 6 July 2021. Published online: 10 July 2021.)

(C) 2021 the author. This is an open access article under the CC BY (International 4.0) license (www.creativecommons.org/licenses/by/4.0/).

\begin{abstract}
In the present paper, new lower and upper bounds on energy and Randić energy of non-singular (bipartite) graphs are reported. Additionally, it is shown that the obtained lower bounds are stronger than two previously known lower bounds in the literature.
\end{abstract}

Keywords: graph energy; Randić energy; bound.

2020 Mathematics Subject Classification: 05C50, 05C90.

\section{Introduction}

Let $G$ be a simple connected graph. Denote by $n$ and $m$ the number of vertices and edges of $G$, respectively. Let $V(G)=$ $\left\{v_{1}, v_{2}, \ldots, v_{n}\right\}$ be the set of the vertices of $G$ and $d_{i}$ be the degree of the vertex $v_{i} \in V(G), i=1,2, \ldots, n$. If $v_{i}$ and $v_{j}$ are two adjacent vertices of $G$, then it is denoted by $i \sim j$. Let $\Delta$ and $\delta$ be the maximum and minimum vertex degrees of $G$, respectively.

Let us denote by $\mathbf{A}=\mathbf{A}(G)$ the adjacency matrix of a graph $G$. The eigenvalues $\lambda_{1} \geq \lambda_{2} \geq \cdots \geq \lambda_{n}$ of $\mathbf{A}$ represent the eigenvalues of $G$ [6]. As well known in spectral graph theory, $\lambda_{1}$ is the spectral radius of $G$ and [6]

$$
\sum_{i=1}^{n} \lambda_{i}=0, \sum_{i=1}^{n} \lambda_{i}^{2}=2 m \text { and } \prod_{i=1}^{n} \lambda_{i}=\operatorname{det} \mathbf{A} .
$$

A graph $G$ is called as non-singular if no eigenvalue of $G$ is equal to zero. For non-singular graphs, it is obvious that $\operatorname{det} \mathbf{A} \neq 0$. A graph $G$ is singular if at least one of its eigenvalue is equal to zero. Then, $\operatorname{det} \mathbf{A}=0$.

The energy of a graph $G$ was defined in [12] as

$$
E=E(G)=\sum_{i=1}^{n}\left|\lambda_{i}\right| .
$$

This graph invariant is utilized to estimate the total $\pi$-electron energy of a molecule represented by a (molecular) graph. [13,22]. A vast literature exists on $E(G)$, for survey and comprehensive information, see [2,11,14,19, 23].

Recently, energy of non-singular graphs has also been studied in the literature. In [8], Das et al. obtained a lower bound on energy of non-singular graphs that improves the lower bounds in [3,22], under certain conditions. Gutman and Das [15] established upper bounds on energy of non-singular (bipartite) molecular graphs. In [15], it was also stated that the upper bound obtained on energy of non-singular molecular graphs improves the upper bound in [3].

The following upper bound on $E(G)$ was found in [11]

$$
E(G) \leq \sqrt{2 m(n-1)+n|\operatorname{det} \mathbf{A}|^{2 / n}} .
$$

The Randić matrix $\mathbf{R}=\mathbf{R}(G)$ of a graph $G$ is defined so that its $(i, j)$ - th entry is equal to $1 / \sqrt{d_{i} d_{j}}$ if $i \sim j$ and is equal to 0 otherwise [1]. The eigenvalues $\rho_{1} \geq \rho_{2} \geq \cdots \geq \rho_{n}$ of $\mathbf{R}$ are called as the Randić eigenvalues of $G$ [1]. Some well known results concerning the Randić eigenvalues are $[1,16]$

$$
\sum_{i=1}^{n} \rho_{i}=0, \sum_{i=1}^{n} \rho_{i}^{2}=2 R_{-1} \text { and } \prod_{i=1}^{n} \rho_{i}=\operatorname{det} \mathbf{R}
$$

\footnotetext{
${ }^{*}$ E-mail address: srf_burcu_bozkurt@hotmail.com
} 
where

$$
R_{-1}=R_{-1}(G)=\sum_{i \sim j} \frac{1}{d_{i} d_{j}}
$$

is the general Randić index of the graph $G[4,18]$.

In full analogous manner with the graph energy [12], the Randić energy of $G$ was introduced in [1]. It was defined as [1]

$$
R E=R E(G)=\sum_{i=1}^{n}\left|\rho_{i}\right|
$$

For details on the properties and bounds of $R E$, see the recent works $[1,9,10,16,17,20,21,23]$.

The following upper bound on $R E(G)$ was obtained in [17,21]

$$
R E(G) \leq 1+\sqrt{(n-2)\left(2 R_{-1}-1\right)+(n-1)|\operatorname{det} \mathbf{R}|^{2 /(n-1)}} .
$$

In the present paper, we find new lower and upper bounds on energy and Randić energy of non-singular (bipartite) graphs. We also show that our lower bounds are stronger than two previously known lower bounds given in $[7,9,14,17]$.

\section{Lemmas}

We now list some lemmas that will be needed for our main results.

Lemma 2.1. [5] Let $x_{i}>-1$ for $1 \leq i \leq n$. If $\sum_{i=1}^{n} x_{i}=0$ and $\sum_{i=1}^{n} x_{i}^{2} \geq a^{2}\left(1-n^{-1}\right)$, then

$$
\sum_{i=1}^{n} \ln \left(1+x_{i}\right) \leq \ln \left(1+a-a n^{-1}\right)+(n-1) \ln \left(1-a n^{-1}\right) .
$$

Lemma 2.2. $[6,27]$ Let $G$ be a graph with $n$ vertices and maximum vertex degree $\Delta$. Then, for each $i=1,2, \ldots, n$

$$
\left|\lambda_{i}\right| \leq \Delta
$$

Lemma 2.3. [10] Let $G$ be a graph with $n$ vertices and without isolated vertices. Then, for each $i=1,2, \ldots, n$

$$
\delta\left|\rho_{i}\right| \leq\left|\lambda_{i}\right| \leq \Delta\left|\rho_{i}\right|
$$

where $\Delta$ and $\delta$ denote, respectively, the maximum and minimum vertex degrees of $G$.

Lemma 2.4. [10] Let $G$ be a graph with $n$ vertices and without isolated vertices and let $\lambda_{1}$ be its spectral radius. Then

$$
\delta(R E(G)-1) \leq E(G)-\lambda_{1} \leq \Delta(R E(G)-1)
$$

where $\Delta$ and $\delta$ denote, respectively, the maximum and minimum vertex degrees of $G$.

Lemma 2.5. $[6,20]$ For a graph $G$, the Randić spectral radius $\rho_{1}=1$.

Lemma 2.6. Let $G$ be a bipartite graph with $n$ vertices and without isolated vertices and let $\lambda_{1}$ be its spectral radius. Then

$$
\delta(R E(G)-2) \leq E(G)-2 \lambda_{1} \leq \Delta(R E(G)-2)
$$

where $\Delta$ and $\delta$ denote, respectively, the maximum and minimum vertex degrees of $G$.

Proof. Note that $\lambda_{1}=-\lambda_{n}$ and $\rho_{1}=-\rho_{n}$, for bipartite graphs [6]. Then, by taking summation (7) over $i=2,3, \ldots, n-1$ and considering Lemma 2.5 and Equations (2) and (5), one can get the required result.

Lemma 2.7. [16] Let $G$ be a graph with $n$ vertices, adjacency matrix $\mathbf{A}$ and Randic matrix $\mathbf{R}$. If $\mathbf{A}$ has $n_{+}, n_{0}$ and $n_{-}$ positive, zero and negative eigenvalues, respectively $\left(n_{+}+n_{0}+n_{-}=n\right)$, then $\mathbf{R}$ has $n_{+}, n_{0}$ and $n_{-}$positive, zero and negative eigenvalues, respectively.

For a graph $G$ with $n$ vertices, the following relation between the determinants of its adjacency and Randić matrices was also given in [16].

Lemma 2.8. [16] If $G$ is a graph with isolated vertices, then $\operatorname{det} \mathbf{R}=\operatorname{det} \mathbf{A}=0$. If $G$ is a graph without isolated vertices, then

$$
\operatorname{det} \mathbf{R}=\frac{\operatorname{det} \mathbf{A}}{\prod_{i=1}^{n} d_{i}}
$$




\section{Main results}

Theorem 3.1. Let $G$ be a connected non-singular graph with $n \geq 2$ vertices and medges. Then

$$
E(G) \geq n\left(\frac{|\operatorname{det} \mathbf{A}|}{(1+(n-1) b)(1-b)^{n-1}}\right)^{1 / n}
$$

where

$$
b=\left[\frac{2 m n-\left(2 m(n-1)+n|\operatorname{det} \mathbf{A}|^{2 / n}\right)}{(n-1)\left(2 m(n-1)+n|\operatorname{det} \mathbf{A}|^{2 / n}\right)}\right]^{1 / 2} .
$$

Proof. We first recall that $\left|\lambda_{i}\right|>0,1 \leq i \leq n$, for a non-singular graph $G$. Let $r=\frac{E(G)}{n}$ and $x_{i}=\frac{\left|\lambda_{i}\right|}{r}-1$, for $1 \leq i \leq n$. Observe that $x_{i}>-1$. By means of Equations (1)-(3), we also have

$$
\sum_{i=1}^{n} x_{i}=\sum_{i=1}^{n}\left(\frac{\left|\lambda_{i}\right|}{r}-1\right)=\frac{\sum_{i=1}^{n}\left|\lambda_{i}\right|}{r}-n=0
$$

and

$$
\begin{aligned}
\sum_{i=1}^{n} x_{i}^{2} & =\sum_{i=1}^{n}\left(\frac{\left|\lambda_{i}\right|}{r}-1\right)^{2}=\frac{\sum_{i=1}^{n} \lambda_{i}^{2}}{r^{2}}-\frac{2 \sum_{i=1}^{n}\left|\lambda_{i}\right|}{r}+n \\
& =\frac{2 m n^{2}}{(E(G))^{2}}-n \\
& \geq \frac{2 m n^{2}}{2 m(n-1)+n|\operatorname{det} \mathbf{A}|^{2 / n}}-n \\
& =\left(\frac{2 m n^{3}}{(n-1)\left(2 m(n-1)+n|\operatorname{det} \mathbf{A}|^{2 / n}\right)}-\frac{n^{2}}{n-1}\right)\left(1-\frac{1}{n}\right) \\
& =\left(n^{2}\left[\frac{2 m n-\left(2 m(n-1)+n|\operatorname{det} \mathbf{A}|^{2 / n}\right)}{(n-1)\left(2 m(n-1)+n|\operatorname{det} \mathbf{A}|^{2 / n}\right)}\right]\right)\left(1-\frac{1}{n}\right) \\
& =(n b)^{2}\left(1-\frac{1}{n}\right) .
\end{aligned}
$$

From Lemma 2.1, we get that

$$
\sum_{i=1}^{n} \ln \left(\frac{\left|\lambda_{i}\right|}{r}\right) \leq \ln (1+(n-1) b)+(n-1) \ln (1-b)
$$

Hence,

$$
\prod_{i=1}^{n}\left|\lambda_{i}\right| \leq r^{n}(1+(n-1) b)(1-b)^{n-1}
$$

that is,

$$
|\operatorname{det} \mathbf{A}| \leq\left(\frac{E(G)}{n}\right)^{n}(1+(n-1) b)(1-b)^{n-1}
$$

This leads to the lower bound (8).

For a non-singular graph $G$ of order $n$, the following lower bound on $E(G)$ was found in [7,14]

$$
E(G) \geq n(|\operatorname{det} \mathbf{A}|)^{1 / n} .
$$

Remark 3.1. Let $b$ be given by Equation (9). Note that $0 \leq b<1$, since $G$ is connected non-singular graph with $n \geq 2$ vertices and the fact that [11,22]

$$
E(G) \leq \sqrt{2 m(n-1)+n|\operatorname{det} \mathbf{A}|^{2 / n}} \leq \sqrt{2 m n} .
$$

Let

$$
f(x)=(1+(n-1) x)(1-x)^{n-1} .
$$

Note that $f$ is decreasing for $0 \leq x<1$ [25]. Thus, $f(b) \leq f(0)=1$, this implies that the lower bound (8) is stronger than the lower bound (10) for connected non-singular graphs. Further, if $G$ is the graph $K_{2}$, then the equality in (8) holds. 
Theorem 3.2. Let $G$ be a connected non-singular graph with $n \geq 2$ vertices, m edges and maximum vertex degree $\Delta$. Then

$$
E(G) \leq \frac{2 m}{n}+n-1+\Delta \ln \left(\frac{n|\operatorname{det} \mathbf{A}|}{2 m}\right) .
$$

The equality in (11) is achieved for $G \cong K_{n}$.

Proof. At first, recall that the following inequality

$$
x \leq 1+x \ln x,
$$

for $x>0$ [24]. Obviously, $\left|\lambda_{i}\right|>0,1 \leq i \leq n$, for a non-singular graph $G$. Considering these facts with Equation (2), we have

$$
\begin{aligned}
E(G) & =\lambda_{1}+\sum_{i=2}^{n}\left|\lambda_{i}\right| \\
& \leq \lambda_{1}+\sum_{i=2}^{n}\left(1+\left|\lambda_{i}\right| \ln \left|\lambda_{i}\right|\right) \\
& \leq \lambda_{1}+n-1+\Delta \sum_{i=2}^{n} \ln \left|\lambda_{i}\right|, \text { by Lemma } 2.2 \\
& =\lambda_{1}+n-1+\Delta \ln |\operatorname{det} \mathbf{A}|-\Delta \ln \lambda_{1} .
\end{aligned}
$$

Let us consider the function $f(x)$, defined by

$$
f(x)=x-\Delta \ln x .
$$

It is not difficult to see that $f$ is a decreasing function in the interval $1 \leq x \leq \Delta$. Notice that $\lambda_{1} \geq \frac{2 m}{n}[6]$ and $\frac{2 m}{n}$ is the average of the vertex degrees that is inevitably greater than unity for connected (molecular) graphs [15]. These together with Lemma 2.2 imply that $1 \leq \frac{2 m}{n} \leq \lambda_{1} \leq \Delta$. Therefore, we have

$$
f\left(\lambda_{1}\right) \leq f\left(\frac{2 m}{n}\right)=\frac{2 m}{n}-\Delta \ln \left(\frac{2 m}{n}\right) .
$$

Based on this inequality and Equation (12), we obtain the upper bound in (11). Moreover, one can readily check that the equality in (11) is achieved for $G \cong K_{n}$.

Theorem 3.3. Let $G$ be a connected non-singular bipartite graph with $n \geq 2$ vertices, $m$ edges and maximum vertex degree $\Delta$. Then

$$
E(G) \leq \frac{4 m}{n}+n-2+\Delta \ln \left(\frac{n^{2}|\operatorname{det} \mathbf{A}|}{4 m^{2}}\right) .
$$

Proof. Notice that $x \leq 1+x \ln x$, for $x>0$ [24]. Further, $\left|\lambda_{i}\right|>0,1 \leq i \leq n$, for non-singular graphs and $\lambda_{1}=-\lambda_{n}$, for bipartite graphs [6]. Taking into account these with Equation (2), we obtain

$$
\begin{aligned}
E(G) & =2 \lambda_{1}+\sum_{i=2}^{n-1}\left|\lambda_{i}\right| \\
& \leq 2 \lambda_{1}+\sum_{i=2}^{n-1}\left(1+\left|\lambda_{i}\right| \ln \left|\lambda_{i}\right|\right) \\
& \leq 2 \lambda_{1}+n-2+\Delta \sum_{i=2}^{n-1} \ln \left|\lambda_{i}\right|, \text { by Lemma } 2.2 \\
& =2 \lambda_{1}+n-2+\Delta \ln |\operatorname{det} \mathbf{A}|-\Delta \ln \lambda_{1}^{2} .
\end{aligned}
$$

Let

$$
f(x)=2 x-\Delta \ln x^{2} .
$$

It can be readily seen that $f$ is a decreasing function in the interval $1 \leq x \leq \Delta$. Recall from Theorem 3.2 that both $\frac{2 m}{n}$ and $\lambda_{1}$ belong to this interval and $\lambda_{1} \geq \frac{2 m}{n}$ [6]. Thus,

$$
f\left(\lambda_{1}\right) \leq f\left(\frac{2 m}{n}\right)=\frac{4 m}{n}-\Delta \ln \left(\frac{4 m^{2}}{n^{2}}\right) .
$$

Combining this with Equation (14), we get the required result in (13). 
In the next theorem, we give a lower bound on Randić energy of non-singular graphs considering the similar techniques in Theorem 3.1 together with Equations (4)-(6) and Lemmas 2.1, 2.5 and 2.7. Therefore, its proof is omitted.

Theorem 3.4. Let $G$ be a connected non-singular graph with $n \geq 3$ vertices. Then

$$
R E(G) \geq 1+(n-1)\left(\frac{|\operatorname{det} \mathbf{R}|}{(1+(n-2) c)(1-c)^{n-2}}\right)^{1 /(n-1)}
$$

where

$$
c=\left[\frac{(n-1)\left(2 R_{-1}-1\right)-\left((n-2)\left(2 R_{-1}-1\right)+(n-1)(|\operatorname{det} \mathbf{R}|)^{2 /(n-1)}\right)}{(n-2)\left((n-2)\left(2 R_{-1}-1\right)+(n-1)(|\operatorname{det} \mathbf{R}|)^{2 /(n-1)}\right)}\right]^{1 / 2} .
$$

For a (connected) graph $G$ of order $n$, the authors derived that $[9,17]$

$$
R E(G) \geq 1+(n-1)(|\operatorname{det} \mathbf{R}|)^{1 /(n-1)}=1+(n-1)\left(\frac{|\operatorname{det} \mathbf{A}|}{\prod_{i=1}^{n} d_{i}}\right)^{1 /(n-1)} .
$$

Remark 3.2. Let $c$ be defined by Equation (16). Observe that $0 \leq c<1$, since $G$ is connected non-singular graph with $n \geq 3$ vertices and the fact that [17,20,21]

$$
\begin{aligned}
R E(G) & \leq 1+\sqrt{(n-2)\left(2 R_{-1}-1\right)+(n-1)|\operatorname{det} \mathbf{R}|^{2 /(n-1)}} \\
& \leq 1+\sqrt{(n-1)\left(2 R_{-1}-1\right)} .
\end{aligned}
$$

Consider the function $f(x)$ defined as follows

$$
f(x)=(1+(n-2) x)(1-x)^{n-2} .
$$

Notice that $f$ is decreasing for $0 \leq x<1$ [26]. Then $f(c) \leq f(0)=1$. Combining this with Lemma 2.8, we deduce that the lower bound (15) is stronger than the lower bound (17) for connected non-singular graphs. Furthermore, if $G$ is the complete graph $K_{n}$, then the equality in (15) is attained.

Theorem 3.5. Let $G$ be a connected non-singular graph with $n \geq 2$ vertices, $m$ edges, maximum vertex degree $\Delta$ and minimum vertex degree $\delta$. Then

$$
R E(G) \leq 1+\frac{n-1+\Delta \ln \left(\frac{n|\operatorname{det} \mathbf{A}|}{2 m}\right)}{\delta} .
$$

The equality in (18) is achieved for $G \cong K_{n}$.

Proof. According to Lemma 2.4 and Equation (12), we have

$$
\begin{aligned}
R E(G) & \leq 1+\frac{E(G)-\lambda_{1}}{\delta} \\
& \leq 1+\frac{n-1+\Delta\left(\ln |\operatorname{det} \mathbf{A}|-\ln \lambda_{1}\right)}{\delta} .
\end{aligned}
$$

From the above and the fact that $\lambda_{1} \geq \frac{2 m}{n}[6]$, we arrive at

$$
R E(G) \leq 1+\frac{n-1+\Delta\left(\ln |\operatorname{det} \mathbf{A}|-\ln \frac{2 m}{n}\right)}{\delta} .
$$

Hence the upper bound in (18) holds. Moreover, it is elementary to check that the equality in (18) is achieved for $G \cong K_{n}$.

Theorem 3.6. Let $G$ be a connected non-singular bipartite graph with $n \geq 2$ vertices, medges, maximum vertex degree $\Delta$ and minimum vertex degree $\delta$. Then

$$
R E(G) \leq 2+\frac{n-2+\Delta \ln \left(\frac{n^{2}|\operatorname{det} \mathbf{A}|}{4 m^{2}}\right)}{\delta} .
$$

Proof. From Lemma 2.6 and Equation (14), we directly get

$$
R E(G) \leq 2+\frac{E(G)-2 \lambda_{1}}{\delta}
$$




$$
\leq 2+\frac{n-2+\Delta\left(\ln |\operatorname{det} \mathbf{A}|-\ln \lambda_{1}^{2}\right)}{\delta}
$$

Considering this with the lower bound $\lambda_{1} \geq \frac{2 m}{n}$ [6], we obtain

$$
R E(G) \leq 2+\frac{n-2+\Delta\left(\ln |\operatorname{det} \mathbf{A}|-\ln \frac{4 m^{2}}{n^{2}}\right)}{\delta}
$$

which is the upper bound in (19).

Remark 3.3. We finally note that the upper bounds in Equations (11), (13), (18) and (19) can be improved using a lower bound such that $\lambda_{1} \geq \gamma \geq \frac{2 m}{n}$ in Theorems 3.2, 3.3,3.5 and 3.6, respectively.

\section{Acknowledgment}

The author would like to thank the two anonymous referees for their valuable comments and suggestions.

\section{References}

[1] S. B. Bozkurt, A. D. Gungor, I. Gutman, A. S. Cevik, Randić matrix and Randić energy, MATCH Commun. Math. Comput. Chem. 64 (2010) 239-250.

[2] Ş. B. Bozkurt Altındağ, D. Bozkurt, Lower bounds for the energy of (bipartite) graphs, MATCH Commun. Math. Comput. Chem. 77 (2017) 9-14.

[3] G. Caporossi, D. Cvetković, I. Gutman, P. Hansen, Variable neighborhood search for extremal graphs. 2. Finding graphs with extremal energy, J. Chem. Inf. Comput. Sci. 39 (1999) 984-996.

[4] M. Cavers, S. Fallat, S. Kirkland, On the normalized Laplacian energy and the general Randić index $R_{-1}$ of graphs, Linear Algebra Appl. 433 (2010) 172-190.

[5] J. H. E. Cohn, Determinants with elements \pm 1 , J. London Math. Soc. 42 (1967) 436-442.

[6] D. Cvetković, M. Doob, H. Sachs, Spectra of Graphs- Theory and Application, Academic Press, New York, 1980.

[7] D. Cvetković, I. Gutman (Eds.), Selected Topics on Applications of Graph Spectra, Math. Inst., Belgrade, 2011.

[8] K. C. Das, S. A. Mojallal, I. Gutman, Improving McClellands lower bound for energy, MATCH Commun. Math. Comput. Chem. 70 (2013) $663-668$.

[9] K. C. Das, S. Sorgun, On Randić energy of graphs, MATCH Commun. Math. Comput. Chem. 72 (2014) 227-238.

[10] K. C. Das, S. Sorgun, I. Gutman, On Randić energy, MATCH Commun. Math. Comput. Chem. 73 (2015) 81-92.

[11] I. Gutman, Bounds for total $\pi$-electron energy, Chem. Phys. Lett. 24 (1974) 283-285.

[12] I. Gutman, The energy of a graph, Ber. Math. Statist. Sekt. Forschungsz. Graz. 103 (1978) 1-22.

[13] I. Gutman, Topology and stability of conjugated hydrocarbons. The dependence of total $\pi$-electron energy on molecular topology, J. Serb. Chem. Soc. 70 (2005) 441-456.

[14] I. Gutman, On graphs whose energy exceeds the number of vertices, Linear Algebra Appl. 429 (2008) 2670-2677.

[15] I. Gutman, K. C. Das, Estimating the total $\pi$-electron energy, J. Serb. Chem. Soc. 78 (2013) 1925-1933.

[16] I. Gutman, B. Furtula, S. B. Bozkurt, On Randić energy, Linear Algebra Appl. 442 (2014) 50-57.

[17] J. He, Y. Liu, J. Tian, Note on the Randić energy of graphs, Kragujevac J. Math. 42 (2018) 209-215.

[18] X. Li, I. Gutman, Mathematical Aspects of Randic-type Molecular Structure Descriptors, Univ. Kragujevac, Kragujevac, 2006.

[19] X. Li, Y. Shi, I. Gutman, Graph Energy, Springer, New York, 2012.

[20] B. Liu, Y. Huang, J. Feng, A note on the Randić spectral radius, MATCH Commun. Math. Comput. Chem. 68 (2012) $913-916$.

[21] A. D. Maden, New bounds on the normalized Laplacian (Randić) energy, MATCH Commun. Math. Comput. Chem. 79 (2018) 321-330.

[22] B. J. McClelland, Properties of the latent roots of a matrix: The estimation of $\pi$-electron energies, J. Chem. Phys. 54 (1971) $640-643$.

[23] E. I. Milovanović, M. R. Popović, R. M. Stanković, I. Ž. Milovanović, Remark on ordinary and Randić energy of graphs, J. Math. Inequal. 10 (2016) 687-692.

[24] D. S. Mitrinović, Elementary Inequalities, P. Noordhoff, Groningen, 1964.

[25] M. G. Neubauer, An inequality for positive definite matrices with applications to combinatorial matrices, Linear Algebra Appl. 267 (1997) $163-174$.

[26] X. Zhang, A new bound for the complexity of a graph, Util. Math. 67 (2005) 201-203.

[27] P. Zumstein, Comparison of Spectral Methods Through the Adjacency Matrix and the Laplacian of a Graph, Diploma Thesis, ETH Zürich, Zürich, 2005. 